\title{
DIFERENCIAÇÃO CLÍNICA DA BRONCOPNEUMONIA MODERADA E GRAVE EM BEZERROS
}

\section{CLINICAL DIFFERENTIATION OF MODERATE AND SEVERE BRONCHOPNEUMONIA IN CALVES}

\author{
Roberto Calderon Gonçalves ${ }^{1}$ Márcio Rubens Graf Kuchembuck ${ }^{2}$ Paulo Roberto Curi ${ }^{2}$ \\ Simone Biagio Chiacchio ${ }^{2}$ Clóvis Teixeira de Almeida ${ }^{2}$ Alexandre Secorun Borges $^{3}$
}

RESUMO

Com o objetivo de identificar os principais sinais clínicos de bezerros com broncopneumonia e definir a doença nas formas moderada e grave, foram utilizados 48 bezerros divididos em 3 grupos: 10 clinicamente sadios (G1), $18 \mathrm{com}$ broncopneumonia moderada (G2) e 20 com broncopneumonia grave (G3). A classificação dos grupos sadios e doentes (broncopneumonia moderada e grave) obedeceu a critérios clínicos e foi confirmada estatisticamente por Análise de Agrupamento. Os resultados evidenciaram ausência de sinais clínicos compatíveis com doença respiratória e menor escore clínico nos bezerros clinicamente sadios (G1). Os bezerros com broncopneumonia foram identificados pela presença de diversos sinais clínicos. confirmando anormalidades pulmonares. Tosse e reflexo de tosse positivo, aumento de temperatura e freqüencia cardíaca, alteracões dos ruídos traqueobrônquico e broncobronquiolar e inspiração interrompida, apesar de relacionados ao diagnóstico de broncopneumonia, não diferenciaram a intensidade moderada e grave do processo. A freqüência respiratória elevada, a dispnéia mista, a submacicez/macicez, área de silêncio, a crepitação grossa e os roncos e sibilos definiram a gravidade do quadro de broncopneumonia.

Palavras-chave: broncopneumonia, bezerros, sinais clínicos, sons respiratórios

\section{SUMMARY}

To evaluate the main clinical signs of bronchopneumonia in calves and determie the classification as moderate or severe form, 48 calves were divided in 3 groups: 10 clinically healthy animals (G1), 18 animals with moderate bronchopneumonia (G2) and 20 animals with severe bronchopneumonia (G3). The classification of these experimental groups was based on clinical characterization and confirmed by statistical Group Analysis. In the clinically healthy calves (G1), the results revealed absence of clinical signs of respiratory disease and low clinical score. The calves with bronchopneumonia were identified by the association of clinical signs showing pulmonary abnormalities. Cough and positive cough reflex, high corporal temperature and cardiac rate, abnormal tracheobronchial and bronchobronchiolar noises and interrupted inspiration were important in the bronchopneumonia diagnosis but did not differentiated the severe or moderate cases. High respiratory rate, dispnoea, signs of compact parenchyma, silence areas and crackles and whizzels were the most important signs to differentiate the clinical cases of bronchopneumonia.

Key words: bronchopneumonia, calves, clinical signs, respiratory sounds.

\section{INTRODUÇÃO}

As doenças pulmonares nos bovinos podem ser provocadas por agentes químicos, físicos ou biológicos, determinando processos inflamatórios, independente do tipo do agente agressor (RADOSTITS et al., 1995). Em especial nos bezerros, representam problema econômico não só pelo custo do tratamento, mas também pelos problemas advindos do comprometimento do desenvolvimento e da produção futura dos animais (BLUM et al., 1996).

A broncopneumonia é caracterizada por alteração inflamatória de brônquios, bronquíolos e parênquima pulmonar (RADOSTITS et al., 1995), sendo o problema respiratório mais freqüente em bezerros, com incidência da ordem de 8,7\% (TAVERA et $\boldsymbol{a l} .$, 1982) a 9,8\% (CURTIS $\boldsymbol{e t}$ al., 1985), podendo chegar a 15\% (ANDREWS et al., 1981). No Brasil, registraram-se índices de 12,7\% em bezerros criados em regime extensivo (BARROS et al., 1965/66) e de 12,27\% nos atendidos no Hospital Veterinário da FMVZ Botucatu - UNESP

\footnotetext{
${ }^{1}$ Professor do Departamento de Clínica Veterinária, Faculdade de Medicina Veterinária e Zootecnia (FMVZ),Universidade do Estado de São Paulo, (Unesp-Botucatu), Distrito de Rubião Junior, 18618-000, Botucatu - SP. e-mail: calderon@fmvz.unesp.br Autor para correspondência.

${ }^{2}$ Professor do Departamento de Clínica Veterinária, FMVZ/Unesp-Botucatu.

${ }^{3}$ Professor do Departamento de Clínica, Cirurgia e Reprodução Animal, Unesp - Araçatuba. Recebido para publicação em 26.11.99. Aprovado em 02.08.00
} 
(GONÇALVES et al., 1993). Considerando-se que o rebanho bovino nacional é estimado em 154 milhões de cabeças (IBGE, 1994), pode-se entender a importância do diagnóstico precoce e da conduta adequada nas afecções pulmonares.

A visão clínica moderna obriga o médico veterinário a trabalhar com relação custo-benefício, de maneira que, quanto mais acurado e precoce o diagnóstico clínico, menos dispendioso é o tratamento. A análise dos sinais clínicos, relacionados às vias aéreas baixas ou posteriores (BREEZE, 1985) e ao parênquima pulmonar, é fator importante na definição do diagnóstico. $\mathrm{Na}$ inspeção do animal, o aumento da frequiência respiratória, afastada a possibilidade de excitação física ou psíquica (STÖBER, 1993), é forte indício de comprometimento das trocas gasosas (RADOSTITS et al., 1995).

De maneira geral, são indicativos de alterações do aparelho respiratório a observação de tosse e a resposta positiva ao reflexo de tosse, traduzindo comprometimento da traquéia (HINCHCLIFF \& BYRNE, 1991), podendo ser uma das manifestações iniciais da doença (PRINGLE, 1992). O corrimento nasal se relaciona tanto à inflamação local (BELKNAP, 1993; CRANDELL, 1993), como à secreção de origem brônquica e pulmonar (WILSON \& LOFSTEDT, 1990; STÖBER, 1993).

São sinais mais específicos de comprometimento do parênquima pulmonar a observação de dispnéia mista, sons submaciços ou maciços à percussão e a auscultação de áreas aumentadas de ruído traqueobrônquico, broncobronquiolar rude e área de silêncio. A dispnéia mista traduz consolidação pulmonar extensa, a ponto de interferir com as trocas gasosas (PRINGLE, 1992; RADOSTITS et al., 1995). O mesmo se diga em relação ao encontro de submacicez/macicez à percussão, também identificada na presença de inflamação, tumores ou abscessos, que consolidam o parênquima pulmonar (PRINGLE, 1992; STÖBER, 1993).

$\mathrm{O}$ aumento na produção de líquido intersticial inflamatório, decorrente dos processos pneumônicos, conduz melhor os sons produzidos na respiração e explica a auscultação de área aumentada do ruído traqueobrônquico e broncobronquiolar (PRINGLE, 1992; STÖBER, 1993). A área de silêncio indica prejuízo funcional do órgão por obstrução de brônquios, bronquíolos e alvéolos (MAREK \& MÓCSY, 1965; BRAZ, 1981). Para ser notada, essa área precisa estar próxima da parede torácica (KOTLIKOFF \& GILLESPIE, 1984).

A inflamação dos brônquios pode levar à formação de líquido que, dependendo da quantidade e da localização na árvore brônquica, produz sons diferentes à auscultação. Quando a quantidade de líquido é intensa nos brônquios de maior calibre, manifesta-se a crepitação grossa (STÖBER, 1993). A obstrução seqüencial de brônquios de menor calibre leva à inspiração interrompida (KOTLIKOFF \& GILLESPIE, 1983), e a deposição de líquido em bronquíolos induz à crepitação fina (STÖBER, 1993). Quando a quantidade de líquido é grande e localizada em área próxima à parede torácica, observa-se frêmito torácico à palpação. $\mathrm{O}$ acúmulo desSe conteúdo líquido na região da traquéia origina o frêmito traqueal (MAREK \& MÓCSY, 1965; BRAZ, 1981; PRINGLE, 1992; STÖBER, 1993).

A deposição de secreções nas vias aéreas determina modificação no fluxo de ar, provocando vibrações de tons mais graves, denominados roncos (MAREK \& MÓCSY, 1965; KOTLIKOFF \& GILLESPIE, 1983 e 1984), ou tons mais agudos, (LEHRER, 1990; PRINGLE, 1992; STÖBER, 1993), identificando as bronquites ou bronquiolites (WILSON \& LOFSTEDT, 1990; HINCHCLIFF \& BYRNE, 1991; STÖBER, 1993).

Inflamações broncopulmonares mais graves podem se acompanhadas de pleurite (RADOSTITS et al., 1995) e o atrito dos folhetos pleurais inflamados determina roce pleural à auscultação (ROUDEBUSH, 1982; KOTLIKOFF \& GILLESPIE, 1984; CURTIS et al., 1986; LEHRER, 1990; HINCHCLIFF \& BYRNE, 1991; STÖBER, 1993). Em processos necrosantes, como nas pneumonias gangrenosas, pode ser notado o odor expiratório pútrido (WILSON \& LOFSTEDT, 1990; ANDREWS, 1992; REBHUN et al., 1995).

A intensidade da broncopneumonia em bezerros, avaliada por métodos semiológicos de auscultação e percussão, pode ser classificada de acordo com a sintomatologia clínica em moderada e grave (CURRLE, 1985), porém, a restrição operacional do diagnóstico clínico e etiológico, principalmente a campo, e a falta de uniformidade na diferenciação de sua intensidade, dificultam o diagnóstico correto e, conseqüentemente, a terapêutica adequada para diminuir as sequielas do processo (BLUM et al., 1996; PINSENT, 1992).

Na tentativa de facilitar o diagnóstico clínico das broncopneumonias em bezerros, este trabalho teve como objetivo identificar os principais sinais clínicos relacionados à doença, na sua forma moderada e grave.

\section{MATERIAL E MÉTODOS}

Foram incluídos neste estudo 48 bezerros, independentemente de raça e sexo, com idade entre um e seis meses, pertencentes a propriedades rurais da região de Botucatu-SP ou admitidos no Serviço 
de Clínica Veterinária de Grandes Animais do Hospital Veterinário da Faculdade de Medicina Veterinária e Zootecnia de Botucatu - UNESP. Os animais foram submetidos a exame clínico, para pesquisa dos critérios diagnósticos das doenças respiratórias (STÖBER, 1993). A presença ou ausência de tais critérios, na maioria das vezes em associação, estabeleceu ou não o diagnóstico de doença respiratória e diferenciou os animais sadios dos doentes (BRYSON, 1985; WILSON \& LOFSTEDT, 1990; ANDREWS, 1992; PINSENT, 1992; PRINGLE, 1992; RADOSTITS et al., 1995; REBHUN et al., 1995). Na dependência da quantidade dos sinais clínicos encontrados, o processo foi caracterizado como broncopneumonia moderada ou grave (Tabela 1).

A partir do exame clínico, os animais foram arranjados em três grupos experimentais: G1: bezerros clinicamente sadios $(n=10)$; G2: bezerros com broncopneumonia moderada $(n=18)$; G3: bezerros com broncopneumonia grave $(n=20)$. Definidos os grupos experimentais, foram iniciados os estudos estatísticos dos sinais clínicos dos bezerros clinicamente sadios (G1) e portadores de broncopneumonia moderada (G2) e grave (G3). Empregou-se a análise de agrupamento (SNEATH \& SOKAL, 1973) para estudar a associação dos sinais clínicos e validar a diferenciação clínica dos grupos de bezerros sadios e portadores de broncopneumonia moderada e grave.

Tabela 1 - Critérios clínicos para a diferenciação das broncopneumonias (BCP) em moderada e grave.

\begin{tabular}{lcc}
\hline Sinais clínicos & $\begin{array}{c}\text { BCP moderada } \\
\text { ausente/presente } \\
(-)(+)\end{array}$ & $\begin{array}{c}\text { BCP grave* } \\
\text { ausente/presente } \\
(-)\end{array}$ \\
\hline Tosse & $-/+$ & $-/+$ \\
Corrimento nasal & $-/+$ & $-/+$ \\
Dispnéia mista & - & + \\
Frêmito traqueal & - & $-/+$ \\
Frêmito torácico & - & $-/+$ \\
Reflexo de tosse & $-/+$ & $-/+$ \\
Submacicez/Macicez & - & + \\
Ruído traqueobrônquico aumentado & $-/+$ & + \\
Ruído broncobronquiolar aumentado & $-/+$ & + \\
Área de silêncio & - & + \\
Inspiração interrompida & $-/+$ & $-/+$ \\
Crepitação grossa & $-/+$ & + \\
Crepitação fina & $-/+$ & $-/+$ \\
Roncos e sibilos & - & + \\
Roce pleural & - & $-/+$ \\
Odor expiratório pútrido & $-/+$ & $-/+$ \\
Temperatura aumentada & $-/+$ & $-/+$ \\
Freqüência respiratória aumentada & $-/+$ & + \\
Freqüência cardíaca aumentada & $-/+$ & $-/+$ \\
\hline
\end{tabular}

* quanto mais associações de sinais clínicos, maior a gravidade do processo adaptado de STÖBER (1993) e CURRLE (1985).

\section{RESULTADOS E DISCUSSÃO}

Os resultados obtidos na avaliação clínica dos bezerros pertencentes aos três grupos em estudo encontram-se apresentados na tabela 2. A freqüência cardíaca aumentada, observada em dois bezerros do grupo clinicamente sadio, poderia indicar alterações cárdio-circulatórias ou excitação psíquica (STÖBER, 1993). O fato desse sinal aparecer isolado de qualquer outro sinal clínico reforça a hipótese de excitação psíquica e confirma a higidez do grupo de animais clinicamente sadios. O grupo de bezerros doentes caracterizou-se pela maior ocorrência de sinais clínicos que, isolados, ou principalmente em associação, demonstravam distúrbio respiratório com comprometimento broncopulmonar (WILSON \& LOFSTEDT, 1990; ANDREWS, 1992; STÖBER, 1993; REBHUN et al., 1995; RADOSTITS et al., 1995).

A broncopneumonia pode se apresentar com intensidade variável no bovino (WILSON \& LOFSTEDT, 1990) e vários critérios são utilizados para a diferenciação do processo, entre eles os sinais clínicos (CURRLE, 1985) e a evolução fisiopatológica do quadro clínico (LEKEUX, 1994). Neste trabalho, optou-se pela análise da freqüência e da intensidade dos sinais clínicos no momento do exame para classificar as broncopneumonias em moderada e grave.

Tosse e reflexo de tosse positivo, apesar de relacionados ao diagnóstico de broncopneumonia (HINCHCLIFF \& BYRNE, 1991; PRINGLE, 1992), não diferenciaram a intensidade do processo, pois estavam presentes em todos os casos. Em relação ao corrimento nasal, houve associação com tosse e reflexo de tosse em 22 animais, mostrando a origem brônquica da secreção (WILSON \& LOFSTEDT, 1990; STÖBER, 1993), embora sem definir a gravidade do quadro clínico. A temperatura aumentada sempre esteve em associação com o aumento da freqüência cardíaca, tanto na intensidade moderada, quanto na grave, relacionando-se com a "síndrome febre" (BRAZ, 1981; RADOSTITS $\boldsymbol{e t}$ al., 1995). Esses sinais clínicos acompanharam os bezerros com broncopneumonia grave, numa freqüência três vezes maior que a observada nos casos de broncopneumonia moderada.

A frequiência respiratória aumentada é um dos mecanismos fisiológicos de compensação pulmonar, na tentativa de manutenção das trocas gasosas (RADOSTITS et al., 1995). Esse sinal clínico foi extremamente importante 
Tabela 2 - Escore e porcentagem $(\%)$ dos sinais clínicos nos grupos de bezerros clinicamente sadios e portadores de broncopneumonia (BCP) moderada e grave.

\begin{tabular}{lcrrrrr}
\hline Sinais clínicos & \multicolumn{2}{c}{ Sadios } & \multicolumn{2}{c}{ BCP moderada } & \multicolumn{2}{c}{ BCP grave } \\
& escore & $(\%)$ & escore & $(\%)$ & escore & $(\%)$ \\
\hline Tosse & 0 & $(0)$ & 18 & $(100,0)$ & 20 & $(100,0)$ \\
Corrimento nasal & 0 & $(0)$ & 11 & $(61,1)$ & 11 & $(55,0)$ \\
Dispnéia mista & 0 & $(0)$ & 00 & $(0,0)$ & 13 & $(65,0)$ \\
Frêmito traqueal & 0 & $(0)$ & 00 & $(0,0)$ & 00 & $(0,0)$ \\
Frêmito torácico & 0 & $(0)$ & 00 & $(0,0)$ & 00 & $(0,0)$ \\
Reflexo de tosse & 0 & $(0)$ & 18 & $(100,0)$ & 20 & $(100,0)$ \\
Submacicez/Macicez & 0 & $(0)$ & 00 & $(0,0)$ & 07 & $(35,0)$ \\
Ruído traqueobrônquico aumentado & 0 & $(0)$ & 11 & $(61,1)$ & 15 & $(75,0)$ \\
Ruído broncobronquiolar rude & 0 & $(0)$ & 13 & $(72,2)$ & 16 & $(80,0)$ \\
Área de silêncio & 0 & $(0)$ & 00 & $(0,0)$ & 03 & $(15,0)$ \\
Inspiração interrompida & 0 & $(0)$ & 18 & $(100,0)$ & 13 & $(65,0)$ \\
Crepitação grossa & 0 & $(0)$ & 01 & $(5,6)$ & 16 & $(80,0)$ \\
Crepitação fina & 0 & $(0)$ & 00 & $(0,0)$ & 00 & $(0,0)$ \\
Roncos e sibilos & 0 & $(0)$ & 00 & $(0,0)$ & 10 & $(50,0)$ \\
Roce pleural & 0 & $(0)$ & 00 & $(0,0)$ & 02 & $(10,0)$ \\
Odor expiratório pútrido & 0 & $(0)$ & 00 & $(0,0)$ & 00 & $(0,0)$ \\
Temperatura aumentada & 0 & $(0)$ & 03 & $(16,7)$ & 10 & $(50,0)$ \\
Freqüência respiratória aumentada & 0 & $(0)$ & 02 & $(11,1)$ & 16 & $(80,0)$ \\
Frequiência cardíaca aumentada & 2 & $(20)$ & 03 & $(16,7)$ & 10 & $(50,0)$ \\
Escore total/número de bezerros & $2 / 10$ & & $98 / 18$ & & $182 / 20$ & \\
\hline & & & & & & \\
\end{tabular}

têm o mesmo significado clínico, relacionando-se com a quantidade de líquido inflamatório pulmonar (PRINGLE, 1992; STÖBER, 1993) e com a gravidade do processo. Observou-se que no grupo com broncopneumonia grave houve maior número de associações entre esses dois sons do que no grupo com broncopneumonia moderada, mostrando a importância da associação dos sinais clínicos para distinguir a intensidade do processo broncopneumônico. Considerando-se, ainda, que todos os animais dos dois grupos doentes apresentaram um ou outro desses sinais clínicos, pode-se afirmar que a presença de tais achados, por confirmar o quadro parenquimatoso, atesta a im-

na diferenciação da broncopneumonia moderada e grave, pois se apresentou com freqüência sete vezes maior nesse último grupo. Quanto mais grave o processo, menor a eficiência das trocas gasosas e maior a necessidade de utilização dos mecanismos de compensação pulmonar (BEECH, 1991; HINCHCLIFF \& BYRNE, 1991; ANDREWS, 1992; PRINGLE, 1992; RADOSTITS et al., 1995). O número de animais doentes com freqüência respiratória elevada foi maior que o número de animais com temperatura alta e taquicardia, mostrando o valor deste sinal clínico isolado no diagnóstico da broncopneumonia.

A dispnéia mista pode ser considerada um fator divisor entre os grupos de bezerros doentes, pois só foi observada no grupo com broncopneumonia grave, numa freqüência de $65 \%$. Nesses casos, a extensa consolidação pulmonar, caracterizada pela dispnéia mista (PRINGLE, 1992; RADOSTITS $\boldsymbol{e}$ $\boldsymbol{a l} .$, 1995), definiu a gravidade do processo. O mesmo se diga em relação às áreas de submacicez/macicez, observadas apenas nos bezerros com broncopneumonia grave, na proporção de $35 \%$. Essa observação sugere que o tecido inflamado estava próximo da parede torácica, com extensão mínima de $4 \mathrm{~cm}$ (PRINGLE, 1992), ratificando a gravidade do processo broncopneumônico pela dimensão da área (RADOSTITS $\boldsymbol{e t}$ al., 1995; REBHUN $\boldsymbol{e t}$ al., 1995).

O aumento da área de ruído traqueobrônquico e a presença de ruído broncobronquiolar rude portância da auscultação pulmonar no diagnóstico das broncopneumonias.

Relacionam-se também à produção aumentada de líquido inflamatório, a área de silêncio, a inspiração interrompida, as crepitações grossa e fina, os roncos e sibilos. A área de silêncio, presente apenas nos casos graves, identificou um pequeno número de animais com preenchimento de brônquios e parênquima pulmonar por líquido de natureza inflamatória, impedindo a passagem de ar e consequiente vibração das paredes brônquicas (MAREK \& MÓCSY, 1965; BRAZ, 1981;).

A inspiração interrompida foi diagnosticada em todos os casos de broncopneumonia moderada e na maioria dos casos graves, denotando presença de líquido, com obstrução seqüencial de brônquios (KOTLIKOFF \& GILLESPIE, 1983), evidenciando comprometimento bronquial. As reações inflamatórias levam à saída de líquido ou à alteração das secreções brônquicas para a defesa do órgão (LEID \& POTTER, 1985; LIGGITT, 1985; SWEENEY \& BEECH, 1991), já em processos iniciais, o que explica a auscultação desse som nos dois grupos de animais doentes. Apesar da importância diagnóstica, ela não serviu para diferenciar a intensidade do processo.

A crepitação grossa e a presença de roncos e sibilos definiram a gravidade das broncopneumonias em bezerros, pois se manifestaram na maioria dos casos graves. Crepitação grossa indica gran- 
de quantidade de líquido nos brônquios, e os roncos e sibilos, concreções de muco aderidas às paredes broncobronquiolares (MAREK \& MÓCSY, 1965; BRAZ, 1981; KOTLIKOFF \& GILLESPIE, 1983 e 1984; LEHRER, 1990; STÖBER, 1993). Portanto, esses sons refletem bronquite intensa e a gravidade do quadro clínico, sendo fundamentais no diagnóstico diferencial dos processos broncopneumônicos.

Casos mais graves de broncopneumonia podem ser acompanhados por pleurite (RADOSTITS et al., 1995). Uma de suas manifestações clínicas é o roce pleural (ROUDEBUSH, 1982; KOTLIKOFF \& GILLESPIE, 1984; CURTIS $\boldsymbol{e}$ t al., 1986; LEHRER, 1990; HINCHCLIFF \& BYRNE, 1991; STÖBER, 1993), que, embora presente em apenas dois casos de bezerros doentes, caracterizou a gravidade do comprometimento pulmonar.

Alguns sinais clínicos, também sensíveis no diagnóstico de processos parenquimatosos e bronquiais, não foram observados nos animais doentes. Frêmitos traqueal e torácico são sinais palpatórios que podem não ter sido diagnosticados nos bezerros doentes, porque dependem da proximidade da área acometida com a parede torácica, da extensão do processo e da quantidade de líquido presente (MAREK \& MÓCSY, 1965; BRAZ, 1981; PRINGLE, 1992; STÖBER, 1993). Crepitação fina, auscultada em edema pulmonar e durante as fases iniciais ou de resolução das pneumonias (STÖBER, 1993), não foi evidenciada no grupo de animais doentes. Esse fato pode ser atribuído à fase intermediária da evolução do processo em que os animais foram examinados ou à dificuldade de auscultação quando esse sinal está associado a outros de maior intensidade. $\mathrm{O}$ odor expiratório pútrido se manifesta nos casos de processos necrosantes, como nas pneumonias gangrenosas (WILSON \& LOFSTEDT, 1990; ANDREWS, 1992; REBHUN et al., 1995). Esse sinal clínico não foi observado em nenhum animal dos grupos doentes.

$\mathrm{O}$ cuidado na diferenciação dos bezerros clinicamente sadios e doentes, e entre estes, os portadores de broncopneumonia moderada e grave, levou à associação de métodos estatísticos para ratificar o diagnóstico clínico. Para que a impressão clínica não fosse subjetiva, utilizou-se a análise de agrupamento (SNEATH \& SOKAL, 1973) e o resultado estatístico quantitativo confirmou os achados do exame clínico dos animais (Figura 1).

Atribuindo-se valor zero para os sinais clínicos ausentes e valor um para os presentes, observou-se que o escore total do grupo de bezerros clinicamente sadios foi 2 , em decorrência do aumento da frequiência cardíaca, assinalado em dois animais. Em contrapartida, o escore foi progressivo entre os animais doentes: 98 para o grupo de bezerros com broncopneumonia moderada e 182 para o grupo com broncopneumonia grave.

A discriminação dos valores atribuídos evidenciou a importância de alguns sinais clínicos na caracterização da intensidade moderada e grave do processo. Contribuíram para atestar a gravidade da broncopneumonia em bezerros: a dispnéia mista (13/182), a submacicez/macicez (7/182), a área de silêncio (3/182), a crepitação grossa (16/182) e os roncos e sibilos (10/182). Inspiração interrompida e os ruídos traqueobrônquico aumentado e broncobronquiolar rude, cujos valores tiveram peso considerável no escore total dos dois grupos doentes, serviram para confirmar o diagnóstico de broncopneumonia, mas não diferenciaram a intensidade do quadro clínico. Da mesma forma, os valores positivos de temperatura e freqüência cardíaca elevadas e, principalmente, o aumento da freqüência respiratória, tiveram sua importância no diagnóstico da doença. O índice progressivo desses dados no escore total dos grupos doentes definiu a intensidade moderada e grave dos bezerros com broncopneumonia.

A ausência ou a associação dos sinais clínicos definiu os três grupos de animais, separando os bezerros sadios dos portadores de broncopneumonia moderada e grave. A análise de agrupamento validou, de forma estatística, a constituição dos três grupos estudados.

\section{CONCLUSÕES}

A presença ou a associação de determinados sinais clínicos confirmou o diagnóstico e caracterizou a intensidade moderada e grave do processo broncopneumônico, sendo que a tosse e o reflexo de tosse positivo, aumento de temperatura e freqüência cardíaca, alterações dos ruídos traqueobrônquico e broncobronquiolar e inspiração interrompida, apesar de relacionados ao diagnóstico de broncopneumonia, não diferenciaram a intensidade moderada e grave do processo.

Além da frequiência respiratória elevada, a dispnéia mista, a submacicez/macicez, a área de silêncio, a crepitação grossa e os roncos e sibilos definiram a gravidade do quadro de broncopneumonia. Os resultados clínicos foram ratificados pela análise estatística: maior escore de sinais positivos nos animais doentes, sendo progressivo entre os portadores de broncopneumonia moderada e grave. 


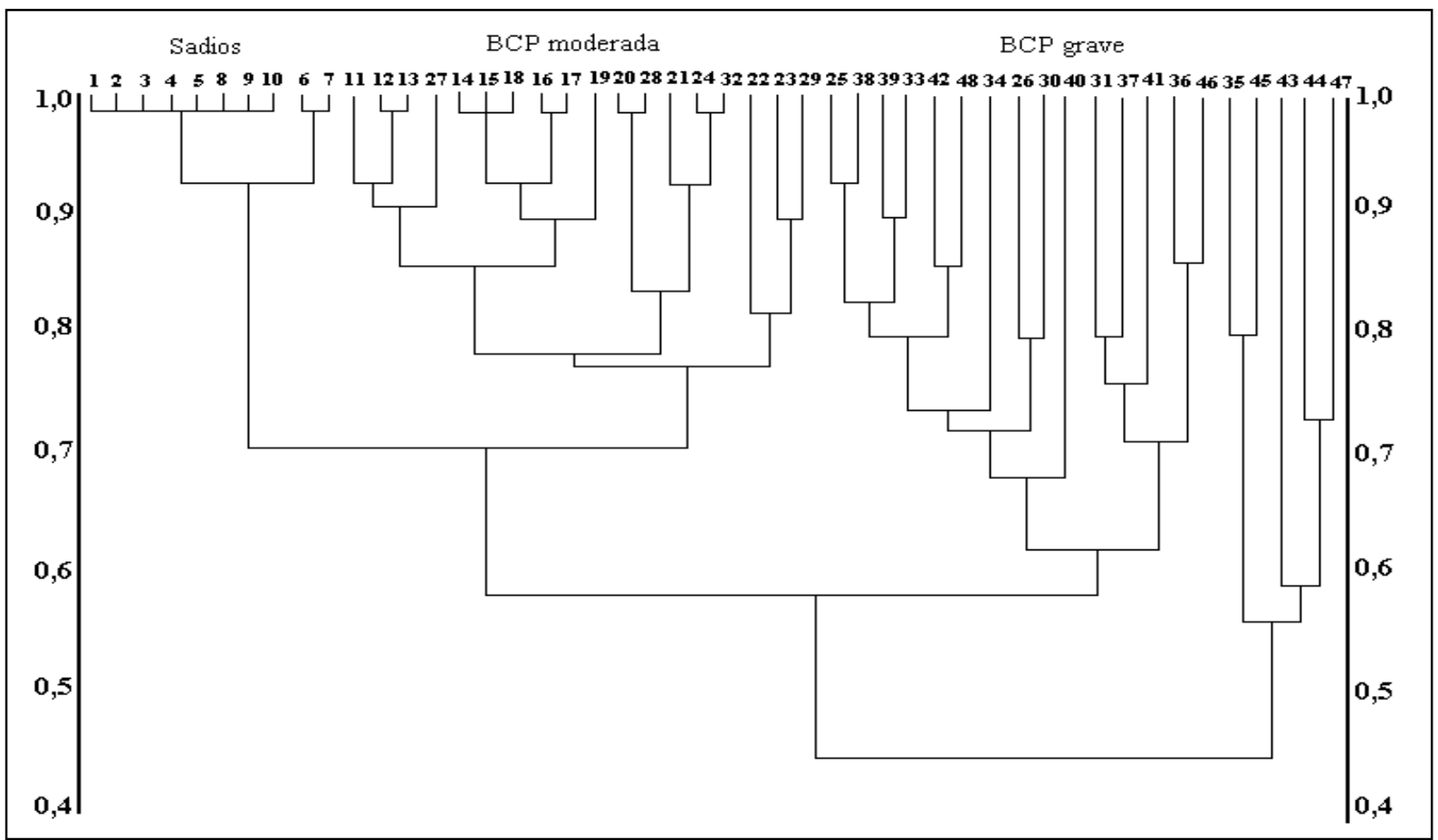

Figura 1 - Análise de agrupamento discriminativa dos bezerros clinicamente sadios (G1) e portadores de brancopneumonia (BCP) moderada (G2) e grave (G3).

\section{REFERÊNCIAS BIBLIOGRÁFICAS}

ANDREWS, A.H. Other conditions. In: ANDREWS, A.H., BLOWEY, R.W., BOYD, R.G., et al. (Eds). Bovine medicine: diseases and husbandry of cattle. Oxford : Blackwell, 1992. Cap.48, p.759-767.

ANDREWS, A.H., PRITCHARD, D.G., MORZARIA, S.P., et al. Observations on a respiratory disease outbreak in weaned suckled calves. Veterinary Record, v.108, p.130-142, 1981.

BARROS, H.M., LAMOUNIER, R.D., ARAUJO, L.M., et al. "Causa mortis" em bezerros Bos indicus, em regime de criação extensiva. Boletim Indústria Animal, v.23, p.199, 1965/66.

BEECH, J. Equine respiratory disorders. Malven : Lea \& Febiger, 1991. Cap.2: Examination of the respiratory tract. p. $27-40$.

BELKNAP, E.B. Recognizing the clinical signs of BRSV infection. Veterinary Medicine, v.88, p.886-887, 1993.

BLUM, J.W., BRUCKMAIER, R.M., MOSER, M. Endocrine, metabolic and hematological changes associated with reduced growth performance during chronic pneumonia in calves: a case study. Dtsch Tierärztl Wochenschr, v.103, p.115-116, 1996.

BRAZ, M.P. Semiologia médica animal 2.ed. Lisboa : Fundação Calouste Gulbenkian, 1981. 644p.

BREEZE, R. Structure, function, and metabolism in the lung. Veterinary Clinics North America: Food Animal Practice, v.1, p.219-235, 1985a.
BRYSON, D.G. Calf pneumonia. Veterinary Clinics North America: Food Animal Practice, v.1, p.237-257, 1985.

CRANDELL, R.A. Viral diseases of cattle. In: HOWARD, J.L. Current veterinary therapy 3: food animal practice. 3.ed. Philadelphia : Saunders, 1993. p.417-419.

CURRLE, M. Endoskopische, tracheobronchialse kretzytologische und arterielle Blutgasuntersuchungen bei bronchopneumonie-kranken Rinder. Hannover, 1985. 179p. Inaugural Dissertation (Doctor Medicinae Veterinariae) - Tierarztliche Hochschule Hannover, 1985.

CURTIS, C.R., ERB, H.N., WHITE, M.E. Epidemiologic study of risk factors for calfhood scours and respiratory syndromes in commercial dairy herds. Journal Dairy Science, v.68, Suppl.1, p.198-199, 1985.

CURTIS, R.A., VIEL, L., McGUIRK,S.M., et al. Lung sounds in cattle, horses, sheep, and goats. Canadian Veterinary Journal, v.27, p.170-172, 1986.

GONÇALVES, R.C., LISBOA, J.A.N., SOUZA, M.V., et al Doenças de bezerros. II Pneumonia. Aspectos clínicos e epidemiológicos na região de Botucatu - SP. In: CONGRESSO INTERNACIONAL DE MEDICINA VETERINÁRIA EM LÍNGUA PORTUGUESA, 6, 1993, Salvador. Anais... Salvador : Comite permanente dos congressos internacionais de Medicina Veterinária de Língua Portuguesa, 1993. p.289. 447p.

HINCHCLIFF, K.W., BYRNE, B. Clinical examination of the respiratory system. Veterinary Clinics North America Equine Practice, v.7, p.1-26, 1991. 
IBGE. Aspectos das atividades agropecuária, extração vegetal e pesca: produção animal. Anuário Estatístico do Brasil, v.54, seção 3-62, 1994.

KOTLIKOFF, M.I., GILLESPIE, J.R. Lung sounds in veterinary medicine: Part I. Terminology and mechanisms of sound production. Compendium Continuing Education Practicing Veterinarian, v.5, p.634-644, 1983.

KOTLIKOFF, M.I., GILLESPIE, J.R. Lung sounds in veterinary medicine: Part II. Deriving clinical information from lung sounds. Compendium Continuing Education Practicing Veterinarian, v.6, p.462-467, 1984.

LEHRER, S. Entendendo os sons pulmonares. São Paulo : Roca, 1990. 147p.

LEID, R.W., POTTER, K.A. Inflammation and mediators of lung injury. Veterinary Clinics North America: Food Animal Practice, v.1, p.377-400, 1985.

LEKEUX, P. Síndrome respiratória bovina: uma perspectiva européia. In: SIMPÓSIO INTERNACIONAL SOBRE DOENÇA RESPIRATÓRIA BOVINA: NOVAS DESCOBERTAS TERAPÊUTICAS. CONGRESSO MUNDIAL DE BUIATRIA, 18, 1994, Bolonha. Resumos.. Bolonha : Schering-Plough, 1994. p.7-13. 48p.

LIGGITT, H.D. Defense mechanisms in the bovine lung. Veterinary Clinics North America: Food Animal Practice, v.1, p.347-366, 1985

MAREK, J., MÓCSY, J. Tratado de diagnóstico clínico de las enfermidades internas de los animales domésticos. 3.ed. Barcelona : Labor, 1965. Antecedentes commemorativos. Anamnesis: p.25-26.

PINSENT, P.J.N. Diagnosis and differential diagnosis in the cow. In: ANDREWS, A.H., BOYD, H., BLOWEY, R.W., et al. Bovine medicine: diseases and husbandry of cattle. Oxford : Blackwell , 1992. Cap.9, p.107-133.

PRINGLE, J.K. Assessment of ruminant respiratory system. Veterinary Clinics North America: Food Animal Practice, v.8, p.233-241, 1992.

RADOSTITS, O.M., BLOOD, D.C., GAY, C.C., et $\boldsymbol{a l}$. Veterinary medicine: a textbook of diseases of cattle, sheep, pigs, goats and horses. 8. ed. London : Baillière Tindall, 1995. 1763p.

REBHUN, W.C., GUARD, C., RICHARDS, C.M. Diseases of dairy cattle. Baltimore : Willians \& Wilkins, 1995. The clinical examination: p.1-10.

ROUDEBUSH, P. Lung sounds. Journal American Veterinary Medical Association, v.181, p.122-126, 1982.

SNEATH, P.H.A., SOKAL, R.R. Numerical taxonomy. San Francisco : Freeman, 1973. 450p.

STÖBER, M. Aparelho respiratório. In: DIRKSEN, G., GRÜNDER, H.D., STÖBER, M. Exame clínico dos bovinos. Rio de Janeiro : Guanabara Koogan, 1993. p.139165 .

SWEENEY, C.R., BEECH, J. Bronchoalveolar lavage. In: $\mathrm{BEECH}$, J. Equine respiratory disorders. Pennsylvania : Lea \& Febiger, 1991. Cap.4, p.55-61.

TAVERA, E.T., TAVERA, F.T., LUGO, G.H., et al. Patologia y bacteriologia de pulmones neumónicos de becerros. Veterinaria Mexico, v.13, p.131-140, 1982.

WILSON, J.H., LOFSTEDT, J. Alterations in respiratory function. In: SMITH, B. Large animal internal medicine: diseases of horses, cattle, sheep and goats. St. Louis : C.V., 1990. V.1, p.47-99.

Ciência Rural, v. 31, n. 2, 2001. 